\title{
High-Energy Cosmic Rays from Radio Galaxies
}

\author{
Björn Eichmann ${ }^{1, *}$ \\ ${ }^{1}$ Ruhr Astroparticle and Plasma Physics Center (RAPP Center), Ruhr-Universität Bochum, Institut für Theoretische Physik IV/ Plasma- \\ Astroteilchenphysik, 44780 Bochum, Germany
}

\begin{abstract}
A detailed investigation of radio galaxies has recently stressed these sources as the possible origin of the cosmic rays observed above $3 \mathrm{EeV}$. Here, the relevance of this model at energies below $3 \mathrm{EeV}$ is investigated. It is shown that the average contribution of radio galaxies can accurately explain the observed CR flux between the second knee and the ankle in the case of a strong source evolution. However, the model cannot provide the increasing heaviness and variance at energies $\lesssim 1 \mathrm{EeV}$ of the observed chemical composition. In addition, it is exposed that the resulting variance of the chemical composition at Earth shows also at higher energies a clear disagreement with the observations, indicating that the compositional contributions by Centaurus A and Cygnus A need to be less different.
\end{abstract}

\section{Introduction}

The origin of the High-Energy Cosmic Rays (HECRs) is still one of the great enigmas of modern astrophysics. From observatories like the Pierre Auger Observatory (Auger) and the Telescope Array (TA) experiment at the highest energies as well as KASCADE, KASCADEGrande and a few other detectors at lower energies, there are basically three main observational characteristics, that describe our current knowledge of the HECRs:

(1.) The energy spectrum, which changes at about $0.4 \mathrm{EeV}$ - the so-called second knee - to a steeper power-law distribution with a spectral index of about 3.3 and flattens above about $3 \mathrm{EeV}$ - the socalled ankle — to a spectral index of 2.6 and a sharp flux suppression above about $10^{19.5} \mathrm{eV}$ [1-3].

(2.) The chemical composition, that shows a decrease of the fraction of heavier elements between about $10^{17} \mathrm{eV}$ and $10^{18.3} \mathrm{eV}$ as well as an increase at energies $>10^{18.3} \mathrm{eV}$ [4-7].

(3.) The large-scale arrival direction distribution, that is usually expressed in terms of the multipoles of their spherical harmonics. Here, Auger recently reported a $5 \sigma$ detection of a dipole with an amplitude of $\approx 6.5 \%$ at energies $>8 \mathrm{EeV}$, while higher-order multipoles as well as the multipoles at lower energies are still consistent with isotropy [8].

A likely source candidate of those extremely energetic particles are radio galaxies due to their powerful acceleration sites within the jets, as already noted by Hillas in 1984 [9]. A recent study by Eichmann et al. [10] - hereafter referred to as $\mathrm{E}+18$ - investigated in great detail

*e-mail: eiche@tp4.rub.de the contribution of $\mathrm{CRs}$ above $3 \mathrm{EeV}$, called ultra-highenergy cosmic rays (UHECRs), by radio galaxies. This model comprises three components: (a) a 3D structure of radio galaxies and EGMF within a radius of $120 \mathrm{Mpc}$; (b) a continuous source function (CSF) derived from a luminosity function of radio galaxies for the contributions from beyond $120 \mathrm{Mpc}$; (c) a contribution of the powerful radio galaxy Cygnus $\mathrm{A}$, which is near the magnetic horizon and is expected to deliver the dominant contribution to UHECRs due to its extreme radio power and brightness. All simulations have been carried out with CRPropa3 [11], including deflections inside the Galaxy according to the magnetic field model of Jansson \& Farrar [12]. So, E+18 draw some solid conclusions on the UHECR contribution by radio galaxies:

(i) The average contribution of all radio galaxies cannot explain both spectrum and composition of UHECR.

(ii) The spectrum and composition of UHECR can be explained by the two brightest radio galaxies in the sky: Cygnus A and Centaurus A. Here, Cygnus A needs to provide a solar-like composition, while Centaurus A needs to have a heavy composition, with an iron fraction comparable to protons at a given cosmic-ray energy. Further, both sources need a cosmic ray load significantly above the average of the bulk of radio galaxies.

(iii) Also the anisotropy constraints at energies $>8 \mathrm{EeV}$ are satisfied, if the UHECRs from Cygnus A are significantly deflected by the EGMF.

In this paper, the model of $\mathrm{E}+18$ is used to check additional aspects of the observational data that have not been taken into account so far. First, the Sect. 2 summarizes the physics of the E+18 model. Subsequently, in Sect. 3 the 
best-fit models of E+18 is applied in order to discuss the CR contribution by the CSF below the ankle as well as the variance of the mean logarithm of the mass number.

\section{The E+18 model}

As shown in great detail in $\mathrm{E}+18$, the established correlation between the jet power $Q_{\text {jet }}$ and the extended radio luminosity $P_{151}$ at $151 \mathrm{MHz}$ from Willott et al. [13] gives for the minimal energy condition [14] a good estimate of the cosmic ray luminosity

$$
Q_{\mathrm{cr}} \simeq \frac{4}{7} Q_{\mathrm{jet}}=1.3 \times 10^{42} g_{\mathrm{cr}}\left(\frac{L_{1.1}}{L_{*}}\right)^{6 / 7} \frac{\mathrm{erg}}{\mathrm{s}}
$$

and the maximal rigidity

$$
\hat{R}\left(Q_{\mathrm{cr}}\right)=g_{\mathrm{acc}} \sqrt{Q_{\mathrm{cr}} / c} .
$$

Here, the typical spectral behavior of $P_{v} \propto v^{-0.6}$ is used to obtain the luminosity $L_{1.1}=1.1 \mathrm{GHz} P_{1.1}$ at $1.1 \mathrm{GHz}$ in units of erg/s, and $L_{*} \approx 4.9 \times 10^{40} \mathrm{erg} / \mathrm{s}$ denotes a characteristic luminosity according to Mauch and Sadler [15]. In doing so, we suppose that the total power in CRs is significantly higher than in relativistic electrons. From the uncertain efficiency of converting jet internal energy into observable radio luminosity, as well as the uncertain details of the acceleration process one obtains the dimensionless coefficients

$$
1 \lesssim g_{\text {cr }} \lesssim 50 \text { and } \quad 0.01 \lesssim g_{\text {acc }} \lesssim 0.5
$$

Introducing different nuclei species $i$ with charge number $Z_{i}$ and an abundance $f_{i}$, the total cosmic ray power per charge number yields

$$
Q_{\mathrm{cr}, i} \equiv Q_{\mathrm{cr}}\left(Z_{i}\right)=f_{i} Z_{i} Q_{\mathrm{cr}} / \bar{Z} .
$$

Here, a simple abundance relation $f_{i}=f_{\odot} Z_{i}^{q}$ has been suggested, where $f_{\odot}$ denotes solar abundances, so that the heaviness of the initial composition can be increased by a single parameter $q$. Since $q>2$ already leads to a dominant contribution at Earth by CR nuclei that left the source as an iron nucleus, it is suggested to keep the initial iron abundance below $2 \%$, hence, $0 \leq q \leq 2$.

Apart from the individual treatment of the sources from the catalog of van Velzen et al. [16], the sources beyond a distance of $120 \mathrm{Mpc}$ from Earth, except for Cygnus $\mathrm{A}$, are treated by a CSF in a $1 \mathrm{D}$ simulation. Here, only the impact of cosmic photon targets is taken into account due to the lack of a known EGMF. So, the local CSF, $\Psi_{i, 0}(R)$, is derived as

$\Psi_{i, 0}(R) \equiv \frac{\mathrm{d} N_{\mathrm{cr}}\left(Z_{i}\right)}{\mathrm{d} V \mathrm{~d} R \mathrm{~d} t}=\int_{\check{Q}_{\mathrm{cr}}}^{\hat{Q}_{\mathrm{cr}}} S_{i}\left(R, \hat{R}\left(Q_{\mathrm{cr}}\right), f_{i}\right) \frac{\mathrm{d} N_{\mathrm{RG}}}{\mathrm{d} V \mathrm{~d} Q_{\mathrm{cr}}} \mathrm{d} Q_{\mathrm{cr}}$,

using the local radio luminosity function, $\mathrm{d} N_{\mathrm{RG}} /\left(\mathrm{d} V \mathrm{~d} Q_{\mathrm{cr}}\right)$, by Mauch and Sadler [15] and the CR spectrum, $S_{i}\left(R, \hat{R}\left(Q_{\mathrm{cr}}\right), f_{i}\right)$, of element species $i$ with an abundance $f_{i}$, emitted by a source with total cosmic ray power per charge number, $Q_{\mathrm{cr}, i}$, up to a maximal rigidity $\hat{R}\left(Q_{\mathrm{cr}}\right)$.
The limits of integration are the smallest, $\check{Q}_{\mathrm{cr}}$, respectively largest, $\hat{Q}_{\mathrm{cr}}$, CR powers that need to be considered. To solve the integral analytically, as shown in $\mathrm{E}+18$, a sharp cutoff of the individual source spectra at $\hat{R}\left(Q_{\mathrm{cr}}\right)$ is supposed. The function $\Psi_{i, 0}(R)$ is the local continuous source function as it is derived from a radio luminosity function determined in the local universe $(z<0.3)$. To extend it to larger redshifts, the approximation

$$
\Psi_{i}(R, z) \approx \Psi_{i, 0}(R)(1+z)^{m-1},
$$

is used with a source evolution index $m \in[3,5]$. The analytical solution of the CSF (6) shows a spectral break at a critical rigidity $R_{*}=g_{\mathrm{acc}} \sqrt{g_{\mathrm{cr}} Q_{*} / c} \approx 2 \times 10^{18} g_{\mathrm{acc}} \sqrt{g_{\mathrm{cr}}} \mathrm{V}$. Thus, the spectral behavior of the CSF yields $\Psi_{i}(R<$ $\left.R_{*}, z\right) \propto R^{-a}$, where $a$ denotes the spectral index of the individual sources, and at high rigidities

$$
\Psi_{i}\left(R>R_{*}, z\right) \propto \begin{cases}R^{-3}, & \text { for } a \leq 2, \\ R^{-1-a}, & \text { for } a>2 .\end{cases}
$$

As already stressed in E+18, this spectral behavior impedes an explanation of the CR data above the ankle by the CSF.

\section{New Prospects}

In order to investigate the observational data below the ankle, the simulations of $\mathrm{E}+18$ are performed again including energies down to $0.1 \mathrm{EeV}$. Further, the large compositional gap between oxygen and iron is filled by adding silicon to the initial composition of the sources. However, there is no significant impact of silicon on the previously obtained results.

\subsection{CR Flux above the second knee}

Based on the best-fit scenario from $\mathrm{E}+18$ the impact of the CSF below the ankle is investigated using individual parameter values for Centaurus $\mathrm{A}$ and Cygnus $\mathrm{A}$ and considering the hadronic interaction model of EPOS-LHC. First the influence of the source evolution parameter is analyzed, yielding an accurate explanation of the CR flux above the second knee by the E+18 model in the case of a source evolution index $m \sim 5$. The Fig. 1 shows that only a large value of $m$ provides a spectral contribution by the CSF that is steep enough to describe the observed dip of the flux at the ankle. Otherwise, Centaurus A and Cygnus A need an initial spectral index $a \ll 1.8$ contravening the first-order Fermi acceleration theory, and as a consequence their baryonic load needs to become significantly smaller.

The parameter values from a simple trial and error fitting to the data are given in table 1 and the corresponding CR fluxes are displayed by the red lines in the right Fig. 1. Note, that this result gives rather a proof of principle than the most likely parameter configuration. In addition, it is shown that the average distribution of radio galaxies can provide the same - or even a higher - baryonic load as Centaurus $\mathrm{A}$ or Cygnus $\mathrm{A}$ in the case of a small acceleration efficiency compared to Centaurus A. Further, the 
Table 1. Best-fit parameters using solar abundances for all sources except for Centaurus A.

\begin{tabular}{ccccccccc}
\hline$m$ & $a$ & $\bar{g}_{\mathrm{cr}}$ & $g_{\mathrm{cr}}^{\mathrm{CenA}}$ & $g_{\mathrm{cr}}^{\mathrm{CygA}}$ & $\bar{g}_{\mathrm{acc}}$ & $g_{\mathrm{acc}}^{\mathrm{CenA}}$ & $g_{\mathrm{acc}}^{\mathrm{CygA}}$ & $q^{\mathrm{CenA}}$ \\
\hline 5 & 1.8 & 18 & 25 & 27 & 0.09 & 0.18 & 0.08 & 2 \\
\hline
\end{tabular}

Upper index of the parameter indicates the corresponding source (Centaurus A or Cygnus A), bar on top of the parameter corresponds to all other sources

critical rigidity $R_{*}$ significantly decrease with decreasing $\bar{g}_{\text {acc }}$ leading to a steeper spectral behavior around the ankle as seen in the left Fig. 1. Thus, more detailed parameter studies are needed to give accurate constraints on the CR physics of the CSF.
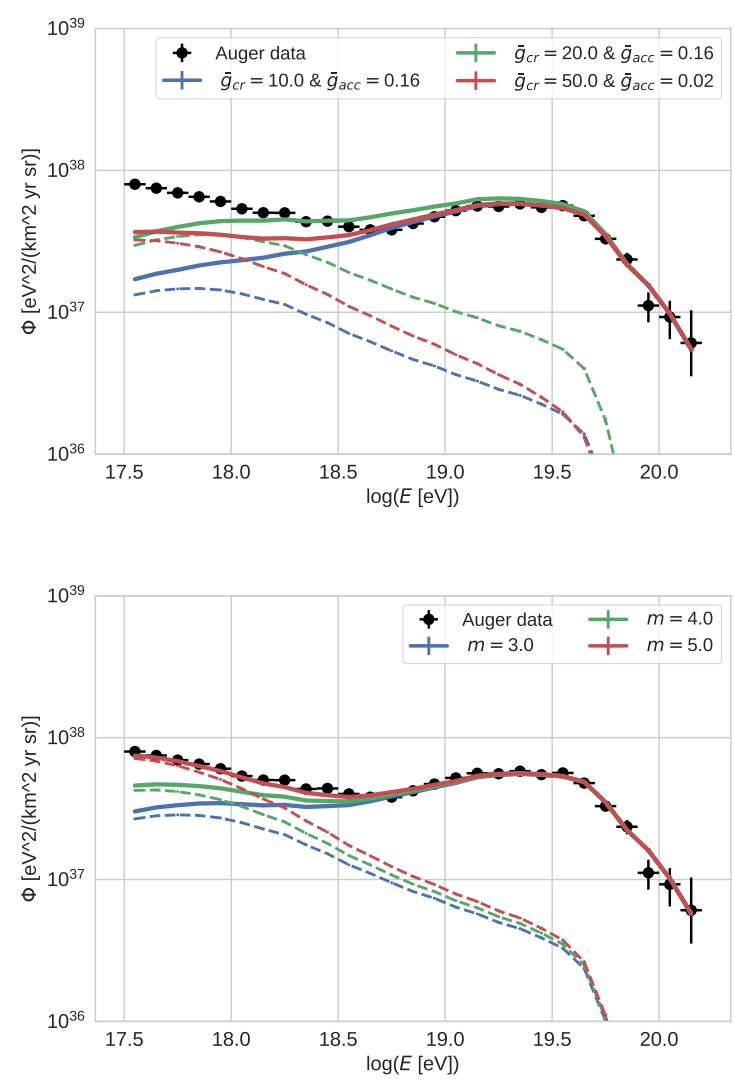

Figure 1. Contribution of the CSF (dashed line) on the total flux (solid line). The left figure shows three different parameter configurations in the case of a source evolution index $m=3$. The right figure displays three different source evolution indexes, while the other parameters are chosen according to table 1 . The observational data is taken from Auger [7].

\subsection{Chemical Composition}

The previously derived fit parameters yield also an excellent agreement with the $\langle\ln A\rangle$ data at energies $>10^{18.2} \mathrm{eV}$, as shown in the left Fig. 2. However, the CSF cannot account for the increase of $\langle\ln A\rangle$ at energies $<10^{18.2} \mathrm{eV}$. Here, another source contribution with a rather heavy composition is needed, as also indicated by the variance of $\ln A$ that is discussed in the following.
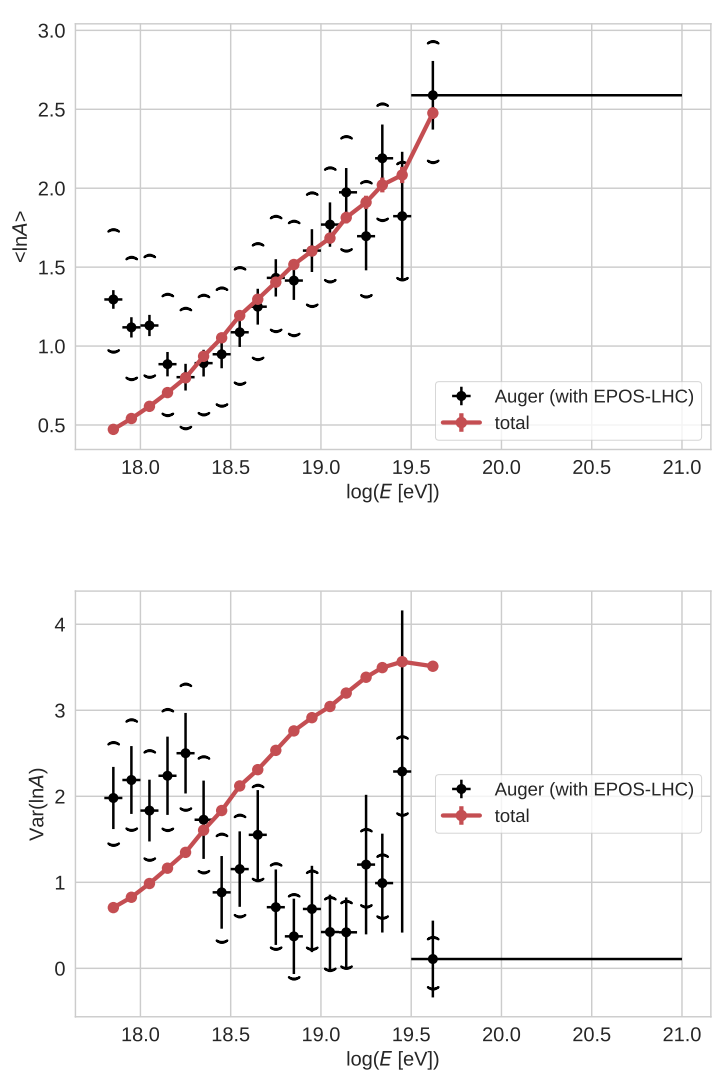

Figure 2. $\langle\ln A\rangle$ (left figure) and $\operatorname{Var}(\ln A)$ (right figure) based on the fit parameters of table 1 . The observational data is taken from Auger [17].

So far, the E+18 model has not been tested with respect to the resulting variance of $\ln A$. There are good reasons, as the observationally derived $\operatorname{Var}(\ln A)$ values need to be treated with some caution, since there are multiple presumptions needed to obtain this observable. Especially an appropriate parametrization of the $X_{\max }$ distribution is needed, which is usually done by generalized Gumbel functions [18]. And note that the hadronic interaction model QGSJetII-04 yields negative $\operatorname{Var}(\ln A)$ values [6], hence, either the QGSJetII-04 model, or the resulting variance analysis needs to be defective.

Keeping this in mind, however, it is clearly shown in the right Fig. 2, that the model cannot explain the observed trend of the $\operatorname{Var}(\ln A)$ values. Basically, the mixing of the strongly differing compositions of Centaurus A and Cygnus A causes too much variance of the chemical composition at Earth. Further, an additional source contribution with a rather heavy initial abundance is needed 
in order to explain the increase in the variance as well as in the mean of the logarithm of the mass number at energies $<10^{18.2} \mathrm{eV}$. Obviously, a solar-like CSF can not do this job. In total, the compositional data by Auger suggest a heavy CR contribution that cuts-off around $1 \mathrm{EeV}$ and a rather pure chemical composition around $10 \mathrm{EeV}$. Both is not provided by the $\mathrm{E}+18$ model so far.

\section{Conclusion}

The inclusion of observational data and simulations below the ankle demonstrates that the CSF for a source evolution index $m \sim 5$ provides an accurate description of the CR flux data between about the second knee and the ankle, whereas Cygnus A and Centaurus A appropriately take over at higher energies. Also the $\langle\ln A\rangle$ data at energies $>10^{18.2} \mathrm{eV}$ is matched perfectly by the model. However, the comparison of the resulting variance of $\ln A$ with observational data shows a clear disagreement and exposes the need for less variance between the initial abundances of Centaurus A and Cygnus A. Up to a certain level, this huge difference is a consequence of the simplified abundance relation, which predominantly yields either a proton or an iron dominated outflow, and can hardly account for the dominance of the CNO group. Further, the current upper limits of other high energy messenger particles like cosmogenic neutrinos show that a strong source evolution, $m \gtrsim 6$ and a proton fraction $f_{p} \gtrsim 0.27$ can be excluded [19]. Thus, the suggested fit scenario is most likely close to these upper limits. So, more detailed investigations are needed that on the one hand, include a generic galactic CR contribution and on the other hand, account for the constraints by the diffuse neutrino and extragalactic gamma-ray background data, as well as the large-scale arrival direction distribution of the CRs.

In total, the $\mathrm{E}+18$ model also provides a promising $\mathrm{CR}$ contribution below the ankle, but the chemical composition clearly indicates the need for an additional heavy CR source at energies $\lesssim 1 \mathrm{EeV}$ - most likely of galactic origin.

\section{References}

[1] D.R. Bergman, J.W. Belz, Journal of Physics G: Nuclear and Particle Physics 34, R359 (2007)
[2] R.U. Abbasi et al. (HiRes), Phys. Rev. Lett. 100, 101101 (2008), astro-ph/0703099

[3] J. Abraham et al. (Pierre Auger), Phys. Lett. B 685, 239 (2010), 1002 . 1975

[4] J. Abraham et al. (Pierre Auger), Phys. Rev. Lett. 104, 091101 (2010), 1002.0699

[5] K.H. Kampert, M. Unger, Astroparticle Physics 35, 660 (2012), 1201.0018

[6] A. Aab et al. (Pierre Auger), Phys. Rev. D90, 122006 (2014), 1409.5083

[7] M. Unger for the Pierre Auger Collaboration (Pierre Auger), PoS ICRC2017, 1102 (2017), 1710. 09478

[8] A. Aab et al. (Pierre Auger), Science 357, 1266 (2017), 1709.07321

[9] A.M. Hillas, Ann. Rev. Astron. Astrophys. 22, 425 (1984)

[10] B. Eichmann, J. Rachen, L. Merten, A. van Vliet, J.B. Tjus, Journal of Cosmology and Astroparticle Physics 2018, 036 (2018)

[11] R. Alves Batista et al., JCAP 1605, 038 (2016), 1603.07142

[12] R. Jansson, G.R. Farrar, Astrophys. J.757, 14 (2012), 1204.3662

[13] C.J. Willott, S. Rawlings, K.M. Blundell, M. Lacy, Mon. Not. Roy. Astron. Soc.309, 1017 (1999), astro-ph/9905388

[14] A.G. Pacholczyk, Radio Astrophysics. Nonthermal Processes in Galactic and Extragalactic Sources (W. H. Freeman \& Co Ltd, San Francisco, 1970)

[15] T. Mauch, E.M. Sadler, Mon. Not. Roy. Astron. Soc.375, 931 (2007), astro-ph/0612018

[16] S. van Velzen, H. Falcke, P. Schellart, N. Nierstenhöfer, K.H. Kampert, Astron. Astrophys.544, A18 (2012), 1206.0031

[17] A. Aab et al. (Pierre Auger), Phys. Rev. D 90, 122005 (2014)

[18] M.D. Domenico, M. Settimo, S. Riggi, E. Bertin, Journal of Cosmology and Astroparticle Physics 2013, 050 (2013)

[19] A. van Vliet, R. Alves Batista, J.R. Hörand el, arXiv e-prints arXiv:1901.01899 (2019), 1901.01899 